\title{
Early visual experience and the receptive-field organization of optic flow processing interneurons in the fly motion pathway
}

\author{
KATJA KARMEIER, RICO TABOR, MARTIN EGELHAAF, AND HOLGER G. KRAPP* \\ Lehrstuhl für Neurobiologie, Fakultät für Biologie, Universität Bielefeld, Postfach 1001 31, D-33501 Bielefeld, Germany \\ (Received March 22, 2000; Accepted August 10, 2000)
}

\begin{abstract}
The distribution of local preferred directions and motion sensitivities within the receptive fields of so-called tangential neurons in the fly visual system was previously found to match optic flow fields as induced by certain self-motions. The complex receptive-field organization of the tangential neurons and the recent evidence showing that the orderly development of the fly's peripheral visual system depends on visual experience led us to investigate whether or not early visual input is required to establish the functional receptive-field properties of such tangential neurons. In electrophysiological investigations of two identified tangential neurons, it turned out that dark-hatched flies which were kept in complete darkness for 2 days develop basically the same receptive-field organization as flies which were raised under seasonal light/dark conditions and were free to move in their cages. We did not find any evidence that the development of the sophisticated receptive-field organization of tangential neurons depends on sensory experience. Instead, the input to the tangential neurons seems to be "hardwired" and the specificity of these cells to optic flow induced during self-motions of the animal may have evolved on a phylogenetical time scale.
\end{abstract}

Keywords: Receptive field, Sensory experience, Optic flow, Visual system, Calliphora

\section{Introduction}

Visual motion is often exploited by biological and technical systems to gain information on the three-dimensional (3D) layout of the environment and the instantaneous rate of self-motion (reviews: Miles \& Wallman, 1993; Barron et al., 1994). Once such information is represented in the nervous system in terms of neuronal activity, it can be processed and transformed into motor signals which allow for course control and gaze stabilization. During the last decades, the way in which optic flow is processed for estimation of self-motion has been intensively investigated. The studies range from human psychophysics (e.g. Warren \& Hannon, 1988), electrophysiological experiments in primates (e.g. Tanaka \& Saito, 1989) and birds (e.g. Wylie et al., 1998), to behavioral as well as electrophysiological investigations in insects (reviews: Hausen \& Egelhaaf, 1989; Egelhaaf \& Warzecha, 1999; Krapp, 2000; Srinivasan \& Zhang, 2000). Most electrophysiological studies have been aimed at identifying the neuronal substrate and the basic processing principles of visual self-motion estimation.

In the third visual neuropil (lobula plate) of the fly, several so-called tangential neurons were found to have receptive-field

*Present Address: Holger G. Krapp is presently located at the Department of Zoology, University of Cambridge, Downing Street, Cambridge CB2 3EY, England.

Address correspondence and reprint requests to: Katja Karmeier, Lehrstuhl für Neurobiologie, Fakultät für Biologie, Universität Bielefeld, Postfach 1001 31, D-33501 Bielefeld, Germany. E-mail: katja.karmeier@ biologie.uni-bielefeld.de properties adapted to sense particular self-motions (Krapp \& Hengstenberg, 1996). They integrate on their extended dendrites the signals of many direction-selective small-field elements. The smallfield elements are arranged in a retinotopic way; that is, neighboring points in the visual space are mapped onto neighboring columns within the visual neuropil (Strausfeld, 1976; Bausenwein \& Fischbach, 1992).

Detailed investigations of the local response properties within the receptive field of several tangential neurons showed that most of these neurons have extended receptive fields with a characteristic spatial distribution of local preferred directions (LPDs) and local motion sensitivities (LMSs) (e.g. Krapp et al., 1998). The resulting response fields in tangential neurons belonging to the vertical system (VS; e.g. Hengstenberg et al., 1982), for instance, show a distribution of LPDs and LMSs which is similar to the distribution of local velocity vectors within optic flow fields induced during rotations around horizontally aligned body axes (Krapp et al., 1998). By comparing the response fields with a matched filter model for sensing particular rotations under certain assumptions, individual VS neurons were shown to indicate the respective self-rotations optimally (Franz \& Krapp, 2000). The appropriate selection and weighting of local motion information thus seems to be one key mechanism underlying self-motion estimation in fly tangential neurons.

The elaborate receptive-field organization of tangential neurons raises the question of whether its orderly development requires visual experience in a similar way as does, for instance, the development of the mammalian visual cortex (e.g. Wiesel, 1982). In flies, recent anatomical studies focus on the effects of different 
light regimes on the development of the animals' visual neuropils. It has been shown that during a critical period of $12 \mathrm{~h}$ after the flies' hatching from pupae, light experience is essential for the neuropils to develop a normal volume. When kept in the dark, the visual neuropils were smaller (Barth et al., 1997). Part of these effects could be attributed to the light-dependent synaptogenesis between photoreceptor cells of the retina and cells of the lamina, the fly's first visual neuropil (Barth et al., 1997; Rybak \& Meinertzhagen, 1997). Lamina cells are most likely input elements of the neuronal circuits processing information about visual motion.

In the present study, we tested in the well-investigated $\mathrm{H} 1$ and V1 tangential neurons (Hausen, 1984) whether visual input is required to develop their characteristic receptive-field organization. $\mathrm{H} 1$ and $\mathrm{V} 1$, rather than other tangential neurons, were chosen for this analysis because of the relative ease with which they can be recorded from extracellularly even for extended periods of time. As for most of the other tangential neurons, H1 picks up local motion signals and-by means of action potentials-conveys the motion information via a thin axon to its telodendritic output ramifications in the contralateral lobula plate. The particular inputoutput organization of this neuron, which is distinctly different from all other spiking tangential neurons investigated so far, allows us to identify $\mathrm{H} 1$ even in experiments using extracellular recording techniques. The latter point holds true for $\mathrm{V} 1$, as well. $\mathrm{V} 1$, however, does not receive direct retinotopic input but is postsynaptic to two or three VS neurons and mediates motion information to the contralateral lobula plate, where its spiking activity can be recorded extracellularly. Thus, the V1 response field reflects the characteristic response fields of VS neurons VS1, VS2 and most likely VS3 (Warzecha, preliminary results, cf. Krapp \& Hengstenberg, 1997 and Krapp et al., 1998) which themselves can only be investigated using intracellular recording techniques.

\section{Methods}

\section{Preparation and electrophysiology}

All experiments were carried out with 2-3 day-old female blowflies (Calliphora) in a dark laboratory at temperatures between 25-30 ${ }^{\circ} \mathrm{C}$. Before the dissection for electrophysiology, the animals were briefly anaesthetized with $\mathrm{CO}_{2}$. Legs and wings were removed and the head was bent ventrally and affixed to a holder. The head was aligned with the horizontal plane of the stimulus device (see below) using the symmetrical deep pseudopupil in the frontal region of both eyes (Franceschini, 1975). The head capsule was opened from behind. Fat tissue, air sacs, and tracheae were removed to get access to the lobula plate. Except the opening in the head capsule, all wounds were closed with wax to prevent the preparation from drying out. Ringer solution $(7.5 \mathrm{~g} \mathrm{NaCl}, 0.14 \mathrm{~g}$ $\mathrm{NaHCO}_{3}, 0.35 \mathrm{~g} \mathrm{KCl}, 2.5 \mathrm{~g}$ glucose, buffered with $0.46 \mathrm{~g} \mathrm{KH}_{2} \mathrm{PO}_{4}$ and $0.59 \mathrm{~g} \mathrm{Na}_{2} \mathrm{HPO}_{4}$ in 11 distilled water resulting in a $\mathrm{pH} \approx 7.1$ ) was used to keep the nervous tissue moist.

$\mathrm{H} 1$ and V1 action potentials were extracellularly recorded from their telodendritic arborizations within the left lobula plate using tungsten electrodes. The electrode tips were sharpened electrolytically and insulated with varnish and had an impedance of about 2 $\mathrm{M} \Omega$. A glass capillary with a broken tip was used as a ground electrode and to supply the brain with Ringer solution. The recorded spikes were amplified using electrophysiological standard equipment, converted into pulses of 2-ms duration, and were sampled at a rate of $0.72 \mathrm{kHz}$ into a $\mathrm{PC}$ by an I/O board (Data
Translation, DT 2801, Marlboro, MA). The software to control the visual stimulus (see below), the data acquisition, and the data analysis was written in ASYST 4.0 (Macmillan Software, La Jolla, CA).

\section{Experimental groups}

In the present work, we did experiments on two different groups of animals. During pupation, the animals were transferred from the stock either to the laboratory where they were kept under seasonal ambient light/dark conditions (control group) or to complete darkness of a darkroom. Flies of the dark group were kept in complete darkness for 2 days after hatching until they were used in the experiment.

The dissection of dark group animals was carried out under red light. The fly photoreceptors (R1-R6) which are involved in visual motion analysis have a maximum absorption at a wavelength of $490 \mathrm{~nm}$. The relative absorbance drops below $5 \%$ for wavelengths greater $590 \mathrm{~nm}$ (Hardie, 1985). Thus, R1-R6 can be expected to be almost insensitive to red light. Correspondingly, the sensitivity of spiking tangential neurons in the lobula plate of Calliphora is reduced to less than $5 \%$ when stimulated with monochromatic motion stimuli at a wavelength of $590 \mathrm{~nm}$ (McCann \& Arnett, 1972). Animals of the control group were dissected under normal microscopy illumination. To reduce the possible influence of uncontrolled visual stimuli while transferring the flies from the preparation place in the darkroom to the experimental setup, animals of the dark group were either transported within a dark cardboard box or their eyes were temporarily covered with nontoxic black paint.

\section{Determining the local response properties}

The local preferred direction (LPD) and local motion sensitivity (LMS) were determined at 46 positions in the right and at four positions within the frontal left hemisphere lying in the region of the binocular overlap. A detailed description of the procedure can be found in Krapp and Hengstenberg (1997). In brief, a black dot $(\varnothing=7.6 \mathrm{deg}$ ) was moved along a circular path $(\varnothing=10.4 \mathrm{deg})$ for 5 cycles first in a clockwise $(\mathrm{CW})$ and then in a counterclockwise (CCW) direction. The speed of the dot's motion was at 2 cycles/s. When the instantaneous direction of dot motion coincided with the local preferred direction of the recorded neuron, its response became maximum (Fig. 1). Comparing the responses to $\mathrm{CW}$ and $\mathrm{CCW}$ motion allowed us to determine the response delay and to correct for it. Subsequently, the phase-locked average of the responses to $\mathrm{CW}$ and $\mathrm{CCW}$ motion can be used to determine the mean angle of the resulting spike distribution by applying circular statistics (Batschelet, 1981). The LPD is given by the mean angle and the LMS is defined as the difference between the response averaged within an interval of \pm 45 deg centered at the LPD and an equally sized interval around the null direction response (cf. black horizontal bars in Fig. 1B). Motion in the null direction is opposite to motion along the preferred direction and usually inhibits the activity of tangential neurons. The LPDs and LMSs are plotted as black arrows in a Mercator map of visual space, within which each position is given by two angles: the azimuth $\varphi$ and the elevation $\theta$ (cf. Figs. 2 and 3). To visualize the global structure of the response fields better, gray arrows were obtained by interpolating between the actually measured data (Matlab routine; Vers. 5.3). The algorithm used is based on Delauny-Triangulation and performs a surface fitting on the present data (Watson, 1994). 
a

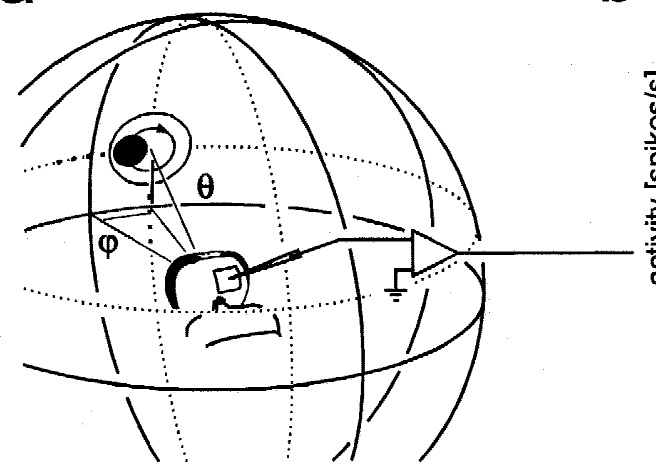

b

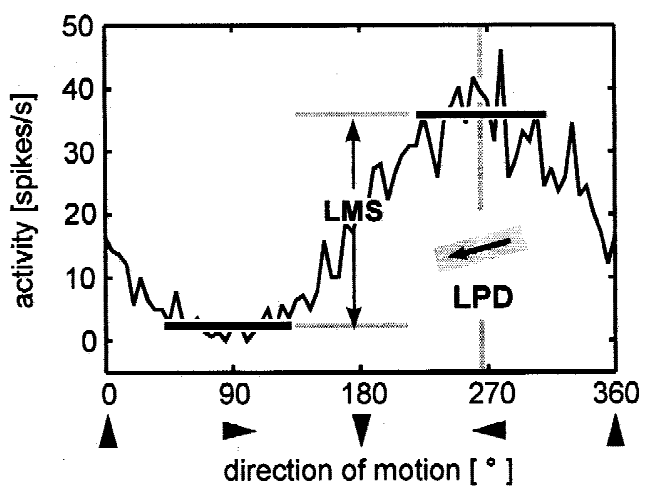

Fig. 1. Determining the local response properties. (A) The fly is centered within an apparatus which allows us to apply a local motion stimulus at many positions $(\varphi, \theta)$ within the visual space. During extracellular H1 and V1 recordings, a black dot travels at 2 circles/s along a circular path, five times in a clockwise and subsequently five times in a counterclockwise direction. Whenever the instantaneous dot motion coincides with the local preferred direction (LPD), the response of the neuron is maximum. Motion in the opposite direction causes maximum inhibition. (B) After phase-locked summation of the response sweeps and correction for the response delay from the resulting circular spike distribution the LPD and LMS can be determined. The LPD is obtained by circular statistics (direction of the mean vector; see black arrow at LPD). The LMS we define by the difference between the mean activity within an interval of \pm 45 deg centered at the LPD (see horizontal black bar centered at about $270 \mathrm{deg}$ ) and the mean activity within an interval of the same size caused by motion in the opposite direction (see horizontal black bar centered at about $90 \mathrm{deg}$ ) (modified from Krapp et al., 1998).
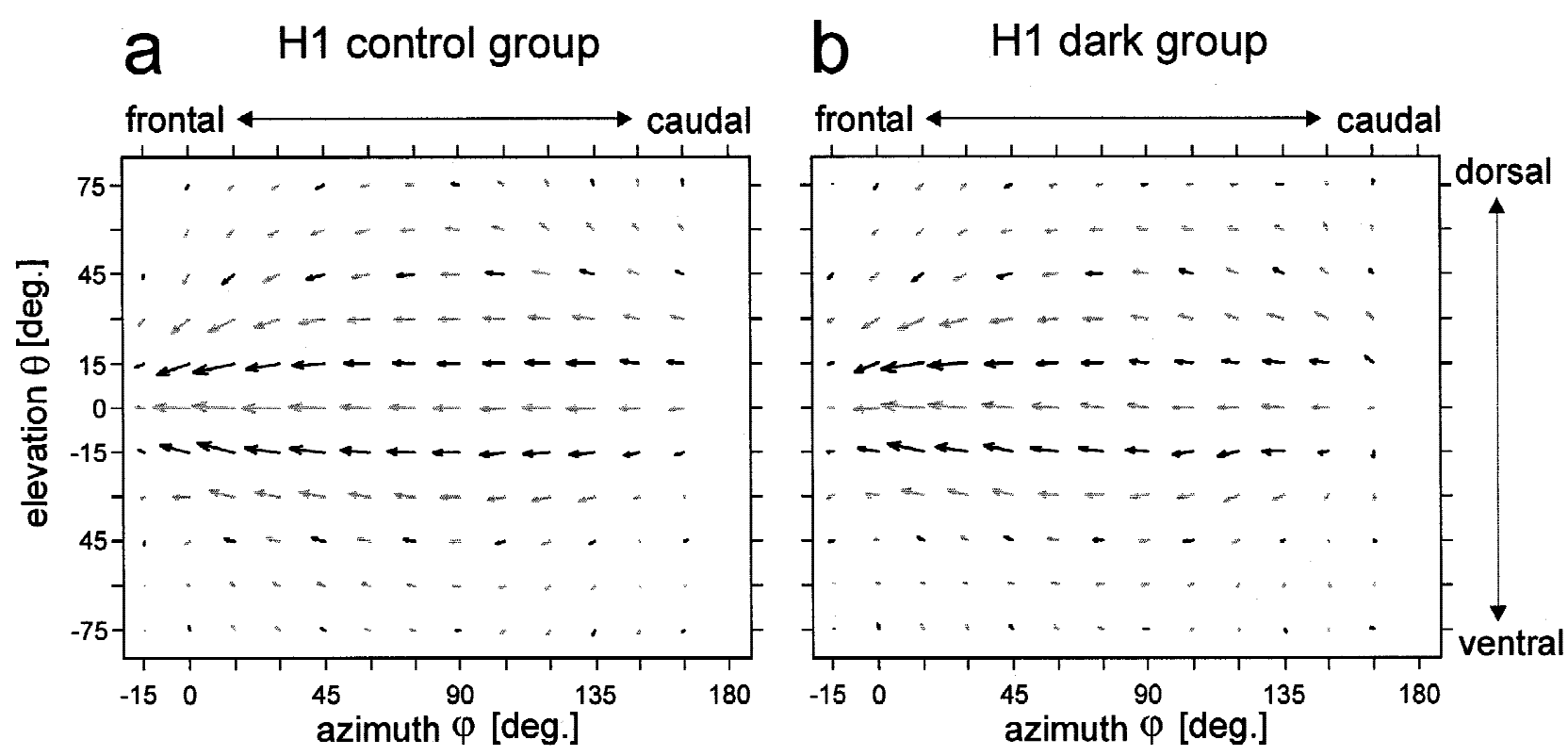

Fig. 2. Mean H1 response field as obtained in five control group animals (A) and five dark group animals (B). Local response properties, that is, local preferred directions (LPDs) and relative local motion sensitivities (LMSs) are shown. The results are plotted along azimuth $\varphi$ and elevation $\theta$ within a map of the right visual hemisphere including the first meridian $(\varphi=-15 \operatorname{deg})$ of the contralateral visual field. The orientation of each arrow gives the LPD and its length indicates the LMS relative to the maximum response measured within the response field. (A) Control group: The H1 neuron responds preferentially to horizontal back-to-front motion along the eye equator. In the frontal and caudal response field above and below the equator, the orientation of the LPDs deviates from the horizontal in a characteristic way; frontodorsally and caudoventrally the LPDs point downwards, whereas ventrofrontally and caudodorsally the LPDs point upwards. H1 is most sensitive in the frontal visual field. Towards the contralateral, dorsal, ventral, and visual field the sensitivity decreases. Along the eye equator the sensitivity is slightly reduced towards the caudal response field. (B) Dark group: The H1 response field of dark-hatched flies kept for about 2 days in complete darkness and were then investigated with respect to their local response properties. The characteristic features of the $\mathrm{H} 1 \mathrm{response}$ field found in flies of the control group are present in the $\mathrm{H} 1$ response field obtained in dark reared animals, as well. 

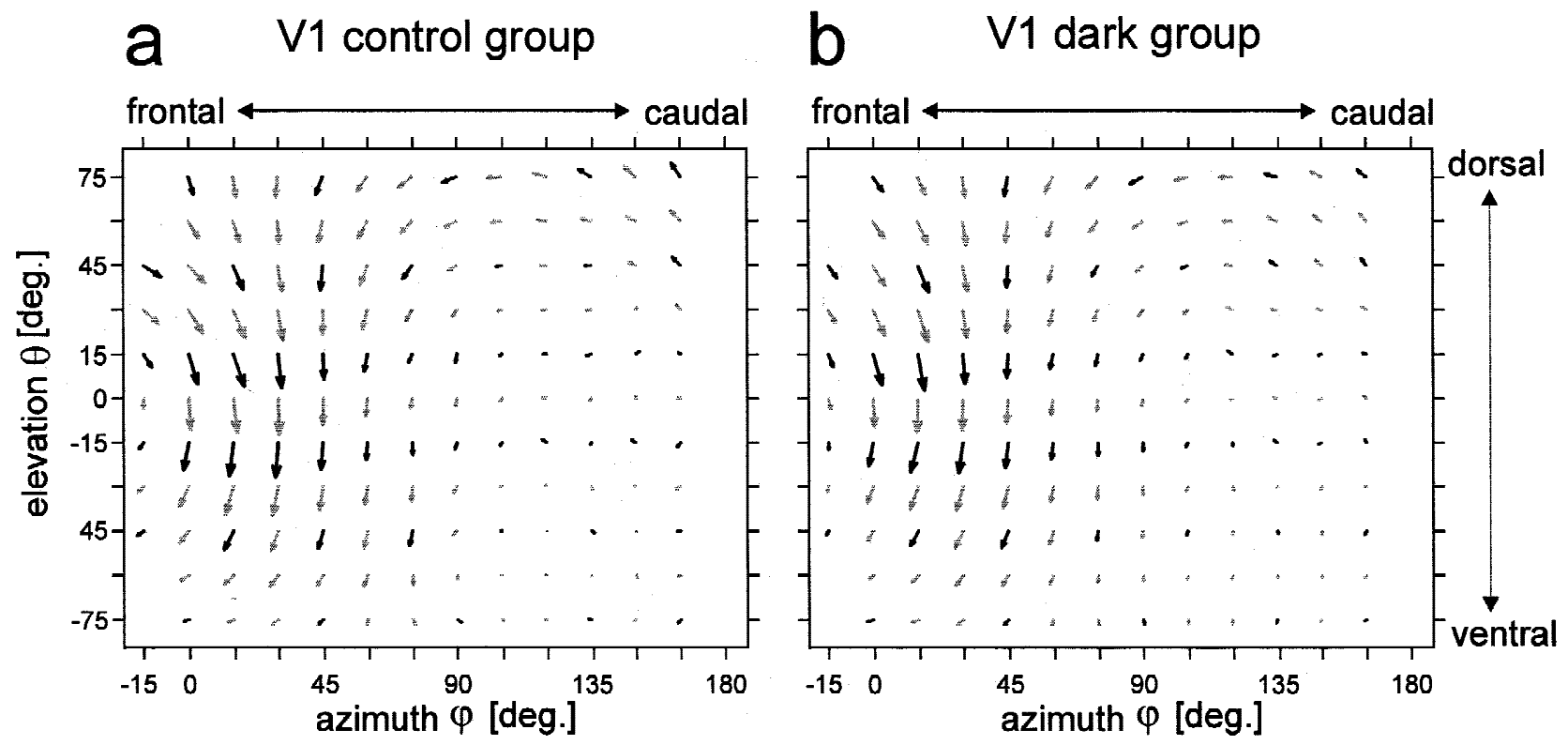

Fig. 3. Mean V1 response field as obtained in two control group animals (A) and six dark group animals (B). Data are plotted in the same way as in Fig. 2. (A) control group: The V1 neuron responds preferentially to vertical downward motion within the equatorial region of the frontal to frontolateral visual field. Although the responses are weak in these areas, in the dorsolateral visual field V1 responds to horizontal and in the dorsocaudal visual field to oblique vertical upward motion. The neuron is insensitive to motion in the equatorial to ventral part of the lateral-to-caudal visual field. The maximum motion sensitivity of $\mathrm{V} 1$ is found in the equatorial region of the frontolateral visual field. V1 is most likely adapted to sense self-rotations around the fly's transverse body axis. (B) dark group: The V1 response field of dark-hatched flies subjected to the same treatment as the H1 dark group. As in case of the H1 neuron, the characteristic fingerprint of the V1 response field observed in flies of the control group is found as well in the V1 response field obtained from dark-reared animals. Note that V1 does not receive retinotopic input but is postsynaptic to two to three VS tangential neurons.

\section{Results}

Response field of the $\mathrm{H} 1$ and V1 neuron in control group and dark group flies

The average $\mathrm{H} 1$ response field obtained in five flies of the control group encompasses almost the entire ipsilateral visual hemisphere (Fig. 2A). Although weakly so, the neuron is still sensitive to motion at the contralateral edge of the frontal region of binocular overlap (azimuth $=-15 \mathrm{deg}$ ). The maximum motion sensitivity is found in the frontal to frontolateral visual field around an elevation of $0 \mathrm{deg}$. Along the eye equator, the LMS decreases slightly towards the caudal part of the visual field. The neuron is only weakly sensitive to motion in the most dorsal and ventral visual field. Generally, H1 responds to horizontal back-to-front motion along the eye equator. Only in the dorsal and ventral parts of the frontal and caudal response fields do the LPDs deviate slightly from the horizontal. These particular features regarding the LPDs as well as the general appearance of the response field confirm the results of a previous investigation (Krapp \& Hengstenberg, 1997).

Fig. 2B shows the average response field obtained in the same number of experiments on dark-reared flies. Except at some positions of small motion sensitivities, the distribution of LPDs and relative LMS within the response field of dark group flies is very similar to that obtained in control group flies (cf. Figs. 2A and 2B).

To estimate the interindividual variability of the LPDs obtained from the two experimental groups, we determined the standard deviations (SD) of the mean LPDs at 24 positions. At these positions, the relative motion sensitivity of both control group and dark group animals was higher than $30 \%$ of the respective maximum motion sensitivity. LPDs at positions with lower motion sensitivity were omitted since their determination was less reliable because of the relatively small numbers of spikes which contributed to the response. The mean of SDs at the 24 positions was determined as measure of the interindividual variability of the LPDs. For the control group animals, the mean $S D$ amounted to $\pm 8 \mathrm{deg}$ and for the dark group animals we found a mean $S D$ of \pm 9 deg. This suggests the interindividual variability of the LPDs to be about the same under the different experimental conditions. To test whether the spatial distribution of LPDs at the 24 positions differ between the control and dark group animals, we performed a two-way ANOVA (e.g. Weber, 1970). The ANOVA did not reveal a significant difference $(\alpha=5 \% ; f(192,1)=1.74)$.

The same comparison was performed on V1 response fields. The average response field obtained in two control group flies is shown in Fig. 3A. Again the general appearance of the control group response field is in very good agreement with the results obtained in an earlier study (Krapp \& Hengstenberg, 1997). In contrast to $\mathrm{H} 1, \mathrm{~V} 1$ is particularly sensitive to vertical downward motion in the equatorial region of the frontal to frontolateral visual field. Besides this, characteristic "fingerprints" of the V1 are its small but sizable sensitivity to horizontal back to front motion in the dorsolateral, and its response to tilted upward motion in the dorsocaudal visual field. All these features are present as well in the average V1 response field of six dark group flies (cf. Figs. 3A and $3 \mathrm{~B}$ ). The local motion sensitivity of the neurons was found to be greater than $30 \%$ of the maximum motion sensitivity in both control group animals and dark group animals at 22 measuring 
positions. The mean SD of the LPDs obtained from these positions amounted to \pm 7 deg in control group animals and \pm 9 deg in dark group animals. Thus-as for the experiments on $\mathrm{H} 1$ - there is no evidence that the interindividual variability is different between the two experimental groups. Moreover, the two-way ANOVA did not show the average V1 response field of dark-reared animals to differ significantly from the one obtained from animals kept under control conditions $(\alpha=5 \% ; f(132,1)=0.98)$.

\section{Absolute local motion sensitivity of the HI and VI neuron in control group and dark group flies}

Within the response fields plotted in Figs. 2 and 3, the LMSs are normalized to the maximum spike rate measured in the respective neuron. In Figs. 4 and 5, the distributions of mean absolute motion sensitivities obtained within the $\mathrm{H} 1$ and V1 response fields of control group and dark group animals are shown in the form of contour plots. Number labels along the contour lines give the LMSs in terms of spikes/s.

The contour plots of the mean absolute motion sensitivities as obtained in H1 neurons of control and dark group animals look very similar. The sensitivity maximum of both groups is close to the fronto-equatorial part of the visual field. From there the sensitivity declines steeply towards the frontal and less steeply towards the other parts of the response field. Within the sensitivity maximum, $\mathrm{H} 1$ neurons of control group animals reach average spike rates of 70 spikes/s (Fig. 4A). Analogously to the analysis of the LPDs, as a measure of the interindividual variability of the motion sensitivity, we calculated the mean $S D$ of the spike rates obtained at the 24 positions. We found a mean SD of \pm 11 spikes $/ \mathrm{s}$ for the control group animals and \pm 9 spikes/s for the dark group animals. Only the peak firing rate of 80 spikes/s is slightly higher in the dark group than in the control group $\mathrm{H} 1$ neurons. To further quantify these results and the motion sensitivity within the two experimental groups, we averaged the absolute LMSs over all measuring positions for each of the five response fields resulting in $29.5 \pm 4.7$ spikes/s for the control group animals and $29.4 \pm 5.1$ spikes/s for the dark group animals. By means of a $t$ test, we could not find significant differences between these two samples $(\alpha>$ $0.4 ; t_{(8)}=0.013$ ). Also the mean spontaneous activity between the two experimental groups was not significantly different (control group: $18.5 \pm 5.1$ spikes/s; dark group: $19.8 \pm 6.3$ spikes $/ \mathrm{s} ; t$ test, $\left.\alpha>0.3, t_{(8)}=0.29\right)$.

The overall distribution of LMSs of V1 obtained in dark group animals looks similar to that derived from control group animals, although differences in the absolute sensitivity are obvious. These differences between the two experimental groups are not accompanied by differences in the mean $S D$ of the spike rate obtained at the 22 measuring positions. In both cases the mean $S D$ amounted to \pm 13 spikes/s. The maximum LMSs in terms of firing rates observed in V1 neurons of the control group animals was on average 60 spikes/s. From its maximum in the equatorial region of the frontolateral visual field, the sensitivity of V1 decreases towards the margins of its response field (Fig. 5A). In contrast, within the area of maximum motion sensitivity, the dark group V1 neurons reached firing rates of up to 100 spikes/s. The high peak firing rate within the response fields of dark group animals results in an averaged overall sensitivity of $38.8 \pm 3.7$ spikes/s as compared with $30.2 \pm 3.5$ spikes/s within the V1 response fields of control group flies. Although weakly significant $\left(\alpha<0.025 ; t_{(6)}=\right.$ 2.84), such a difference is still in the range of variability when different experimental data sets obtained with the same stimuli are compared. The mean spontaneous activity of control group V1 neurons $(23.9+10.0$ spikes/s) was smaller than that of dark group

\section{a $\mathrm{H} 1$ control group}

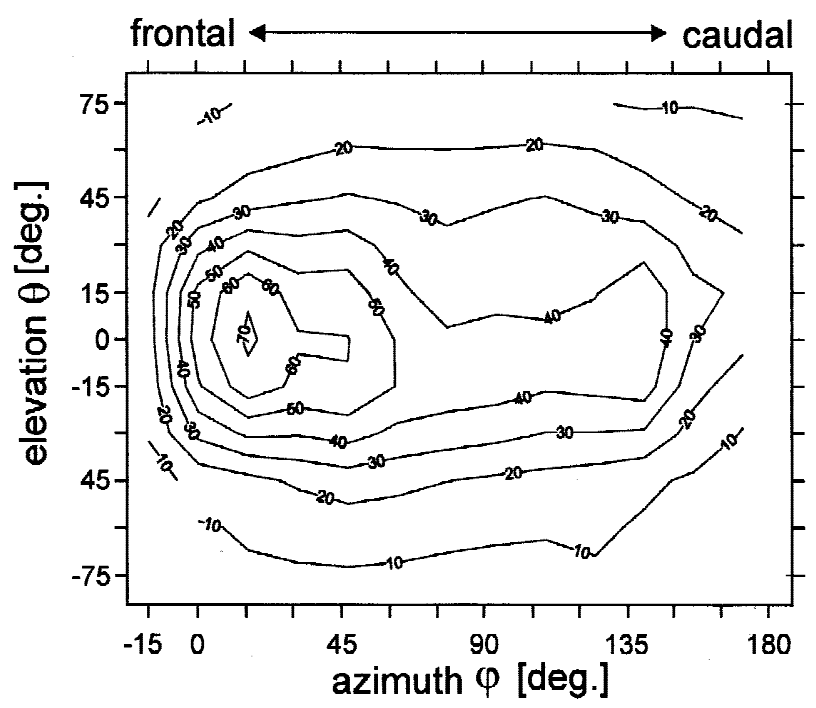

\section{b $\quad$ H1 dark group}

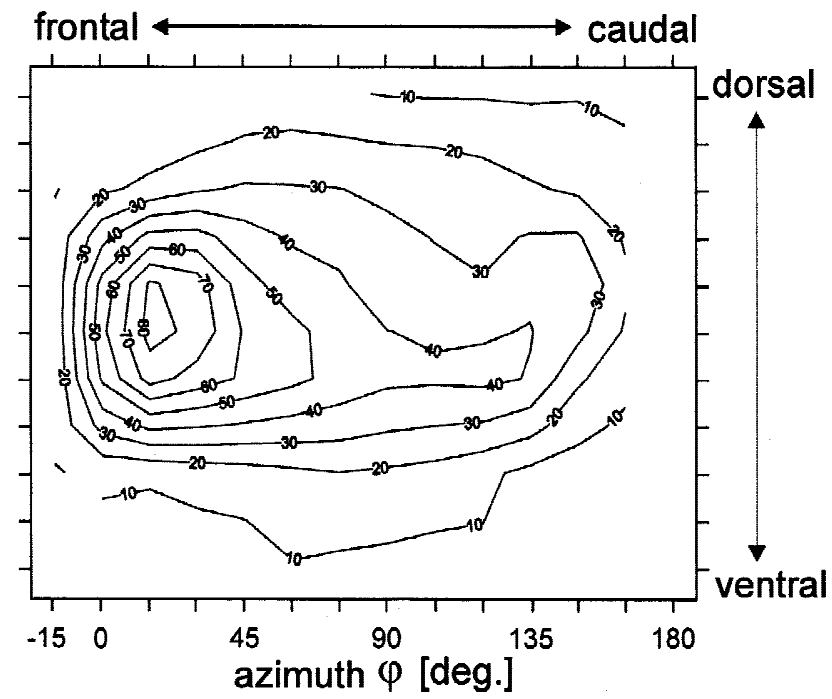

Fig. 4. The absolute motion sensitivity of the $\mathrm{H} 1$ neuron presented by means of contour plots for $\mathrm{H} 1$ response fields obtained in control group flies (A) and for H1 response fields measured in dark group flies (B). Small numbers along the contour lines give the mean absolute motion sensitivity in spikes/s. (A) The maximum motion response in control group H1 neurons amounts to 70 spikes/s. The sensitivity decreases towards the receptive-field margins to less than 10 spikes/s. (B) The contour plot of the absolute motion sensitivities obtained from $\mathrm{H} 1$ neurons of the dark group is very similar to that found in the control group H1 neurons. The maximum response is 80 spikes/s and decreases in about the same way as it does in control group neurons. Same data as shown in Fig. 2. 

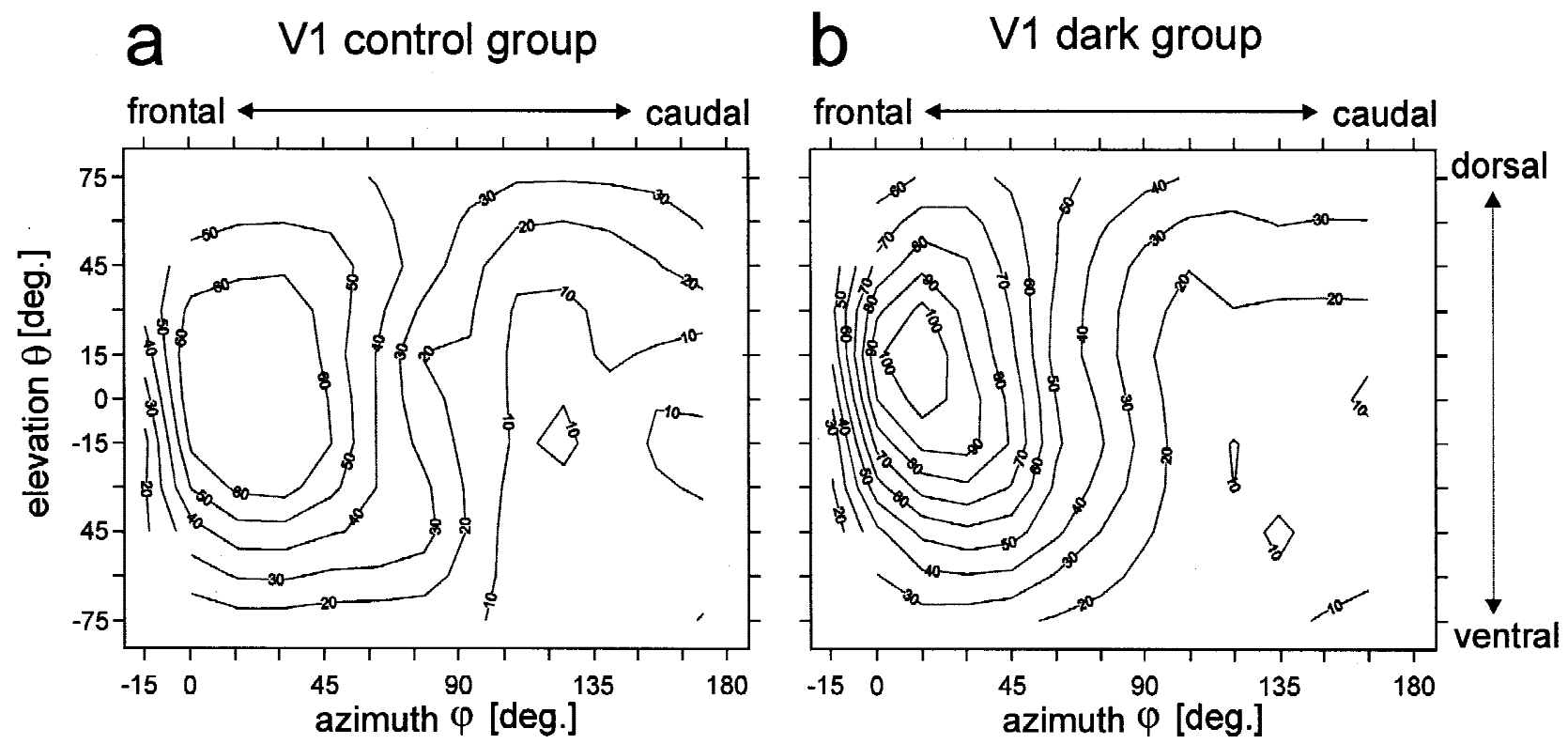

Fig. 5. The absolute motion sensitivity of the V1 neuron presented by contour plots for response fields obtained in control group flies (A) and for dark group flies (B). Data are plotted the same way as in Fig. 4. (A) The maximum motion response in control group V1 neurons amounts to $60 \mathrm{spikes} / \mathrm{s}$ and is broadly tuned within the frontal to frontolateral region of the equatorial visual field. Towards the receptive-field margins the sensitivity drops. (B) The overall appearance of the contour plot representing the absolute V1 sensitivities of dark group flies is similar to that of V1 neurons obtained in control group animals. However, the dark group neurons have a higher sensitivity peak and thus a steeper sensitivity profile in the equatorial region of the frontal to frontolateral visual field. Nevertheless, the decrease in motion sensitivity towards the receptive-field margins is in qualitative accordance with the sensitivity profile obtained for the V1 neurons of the control group flies. Same data as shown in Fig. 3.

cells $(39.9+6.9$ spikes $/ \mathrm{s})$. At significance levels $\alpha<0.025$, the $t$ test showed the mean spontaneous activities of the two groups to be different with respect to this parameter $\left(t_{(6)}=2.60\right)$.

In summary, the results suggest that the overall receptive-field organization of the tangential neurons $\mathrm{H} 1$ and $\mathrm{V} 1$ is basically independent of sensory experience during early posthatching life. Neither the distribution of the local preferred directions nor the relative local motion sensitivities seem to be distinctly affected by rearing the animals in complete darkness. The only indication for sensory experience might be that dark-reared animals are slightly more sensitive to motion than the control animals.

\section{Discussion}

Our experiments suggest that the overall receptive-field organization of the tangential neurons $\mathrm{H} 1$ and $\mathrm{V} 1$ is not dependent on early visual experience. The characteristic distributions of local preferred directions and relative local motion sensitivities within the neurons' receptive fields were found to be the same in control animals and in animals which were reared for about 2 days in complete darkness and were never confronted with any selfmotion induced visual input. Only the absolute sensitivity to motion might be slightly larger in dark-reared flies.

It is critical for these conclusions to be valid that the flies of the dark group did not encounter any uncontrolled visual motion cues which could possibly induce the observed response-field organization. The dark group was raised in a darkroom and its cage was additionally covered with black cloth to completely exclude any motion cues. Dissections for electrophysiology were carried out under red light thereby reducing potential motion stimuli to a minimum (see Methods). We do not expect the still remaining motion cues to be responsible for the development of the response fields. Firstly, the flies did not perceive any self-motion induced motion cues. Secondly, the local motion stimulus approximates all possible directions of motion which makes it inappropriate to imprint a particular distribution of local preferred directions during the experiment. To assess a potential influence of visual motion on the receptive-field organization, we subjected another group of five dark-hatched flies for 2 days to a massive unidirectional motion stimulus within a circular aperture of $60 \mathrm{deg}$ centered within the $\mathrm{H} 1$ neuron's region of maximum motion sensitivity. Even though we chose stimulus parameters which elicit strong responses in both electrophysiological and behavioral experiments, the overall appearance of the H1 response field was not affected. Only the LPDs in the stimulated part of the visual field were weakly but systematically shifted by, on average, $7.5 \mathrm{deg}$ relative to the LPDs of control group flies $\left(\alpha=1 \% ; f_{(80,1)}=33.2\right)$. Even such a strong nonphysiological stimulus induced only a small change in the local preferred directions. Thus, it is unlikely that the inevitable (comparably) weak visual input the dark group flies might have received during their handling prior to the experiment could have affected the characteristic response field organization of $\mathrm{H} 1$ and V1.

\section{Experience-dependent development of the fly visual system}

For Drosophila and Musca anatomical investigations have shown that the posthatching development of the optic lobes depends on the applied light regime (Barth et al., 1997; Rybak \& Mein- 
ertzhagen, 1997). Flies kept in complete darkness were found to develop a first visual neuropil (lamina) reduced in volume by up to $30 \%$ compared with flies raised under normal light conditions. These marked differences were attributed to the absence of a lightdependent size increase of the photoreceptor terminals which, under normal light conditions, is induced in animals within a critical period which is maximal during about the first $12 \mathrm{~h}$ of the fly's adult life (Barth et al., 1997). Electron-microscopic studies have also shown the synaptogenesis of the connections between the photoreceptor cells and their follower neurons (large monopolar cells: LMCs) in the lamina to be affected by light reversals, reducing the synaptic density in light-reared flies subject to dark exposure (Rybak \& Meinertzhagen, 1997). Even though these findings demonstrate a large degree of plasticity of the visual system, the local preferred directions characteristic of the neurons $\mathrm{H} 1$ and V1 seem not to be affected. On the other hand, it might have been expected that the reduction in the size of the peripheral visual neuropils and of the number of synapses found in dark-exposed flies lead to a general sensitivity reduction of the visual system. Although this effect would not necessarily change the local preferred directions, it should decrease the sensitivity to motion. Our experiments suggest, however, that-at least in the V1 neuronthe absolute motion sensitivity is increased (Figs. 5A and 5B). In this context, a study of Kral and Meinertzhagen (1989) on the housefly Musca might be interesting; they investigated the effect of different light regimes on the number of the feedback synapses of one type of LMCs onto the photoreceptor cells. Occluding one eye with black paint led to an increased number of feedback synapses during the first 2-4 days after eclosion compared with the number of synapses supplied by the unoccluded eye. Although the function of the feedback synapses is not entirely clear, they are thought to shape the LMC responses by feeding back their signals to the presynaptic photoreceptor cells (Laughlin \& Osorio, 1989). One could speculate that the increased number of feedback synapses might compensate for a general reduction of the system's activity due to the decreased synaptogenesis between photoreceptors and LMCs in dark-reared animals. Alternatively, it might be possible that, during the electrophysiological measurements, the photoreceptors of dark-reared flies were in a different adaptational state than the photoreceptors of the control group animals. This possibility could be tested by keeping, prior to the experiments, control group animals for a few hours in darkness.

\section{"Hardwiring" particular tasks in visual sensory systems}

Our experimental findings strongly suggest that the input organization of the tangential neurons is genetically fixed, rather than tuned by early visual experience. What could be the rationale for a genetically determined receptive-field organization in neurons concerned with self-motion estimation from optic flow? The specific structure of the environment encountered by the fly in a given situation is unpredictable. The mean distance distribution of the surroundings, however, is most likely to be similar during the lifetime of an individual; that is, distances towards the ground are closer than to the sky. In addition, the fly's repertoire of performed self-motions can also be expected to be similar during its life. Thus, the conditions for estimating self-motion from the momentary optic flow are, on average, relatively stable. In such a case, hardwiring the neuronal machinery in a task-specific way may be the superior adaptation strategy compared with an experiencedependent neuronal plasticity. Moreover, flies need to be able to visually navigate and thus to estimate their self-motion immedi- ately after hatching. If this task were not supported by the neuronal system from the very beginning of their adulthood but required visual experience, the behavioral repertoire-for instance, visual navigation in the context of foraging and predator avoidancewould be rather restricted and the animal's survival would be fairly unlikely.

A similar situation seems to be given in the polarization vision system of the cricket. These animals use, like bees and ants, the pattern of polarized light for navigation purposes. The neuronal key elements involved in the analysis of the polarization pattern are the so-called POL neurons. These visual interneurons selectively integrate the output of many photoreceptors which are tuned to similar e-vector orientations. The resulting response properties, that is, the specificity to a particular polarization pattern, has been found to develop even if the animals never encountered any polarized light at all (Helbling \& Labhart, 1998).

The hypothesis that self-motion estimation is probably genetically determined is indirectly supported by a recent study comparing the fly eye geometry with the distribution of LPDs measured in tangential neurons. The preferred direction of small-field elements is assumed to be determined mostly by interactions between neighboring ommatidia along the rows and columns of the compound eye lattice (Buchner, 1976). It turns out that the orientation of the ommatidial lattice may be sufficient to explain the distribution of the LPDs within response fields of several tangential neurons (Krapp \& Egelhaaf, 1999; Petrowitz et al., 2000). Since the ommatidial lattice is fully developed immediately after hatching, it must be genetically determined. If the LPDs were solely caused by the orientation of the eye lattice, they should therefore also be genetically determined.

In summary, the present study shows that the receptive-field organization of the tangential neurons is basically independent of visual sensory information during early posthatching development. Rather the distributions of LPDs and LMSs in tangential neurons are suggested to be genetically determined and-as a consequence of a task-specific adaptation-to have become established on a phylogenetic time scale.

\section{Acknowledgments}

The authors want to thank A-K. Warzecha, R. Kern, and R. Kurtz for critically reading and discussing the manuscript. We also thank K. Götz and R. Hengstenberg from the Max-Planck-Institut für Biologische Kybernetik, Tübingen, for kindly lending the stimulus equipment. In addition, we would like to thank an anonymous referee for language corrections and comments which significantly helped to improve the manuscript. The project was supported by the Deutsche Forschungsgemeinschaft (DFG).

\section{References}

Barron, J.L., Fleet, D.J. \& Beauchemin, S.S. (1994). Performance of optical flow techniques. International Journal of Computer Vision 12, 43-77.

Barth, M., Hirsch, H.V.B., Meinertzhagen, I.A. \& Heisenberg, M. (1997). Experience-dependent developmental plasticity in the optic lobe of Drosophila melanogaster. Journal of Neuroscience 17, 1493-1504.

Batschelet, E. (1981). Circular Statistics in Biology. London: Academic Press.

Bausenwein, B. \& Fischbach, K.F. (1992). Activity labeling patterns in the medulla of Drosophila melanogaster caused by motion stimuli. Cell and Tissue Research 270, 25-35.

BuCHNER, E. (1976). Elementary movement detectors in an insect visual system. Biological Cybernetics 24, 85-101.

EgelhaAF, M. \& Warzecha, A.-K. (1999). Encoding of motion in real time by the fly visual system. Current Opinion in Neurobiology $\mathbf{9}$, $454-460$. 
Franceschini, N. (1975). Sampling of visual environment by the compound eye of the fly: Fundamentals and applications. In Photoreceptor Optics, ed. Snyder, A.W. \& Menzel, R., pp. 98-125. Berlin, Heidelberg, New York: Springer.

Franz, M.O. \& KrapP, H.G. (2000). Wide-field, motion-sensitive neurons and matched filters for estimating self-motion from optic flow. Biological Cybernetics 83, 185-197.

HARDIE, R.C. (1985). Functional organization of the fly retina. In Progress in Sensory Physiology, Vol. 5, ed. Autrum, H., Ottoson, D., PerL, E.R., Schmidt, R.F., ShimadzU, H. \& Willis, W.D., pp. 1-79. Berlin, Heidelberg, New York, Tokyo: Springer.

Hausen, K. (1984). The lobula-complex of the fly: Structure, function and significance in visual behaviour. In Photoreception and Vision in Invertebrates, ed. ALI, M.A., pp. 523-559. New York, London: Plenum Press.

Hausen, K. \& EgelhaAf, M. (1989). Neural mechanisms of visual course control in insects. In Facets of Vision, ed. Stavenga, D.G. \& Hardie, R.C., pp. 391-424. Berlin, Heidelberg: Springer.

Helbling, H. \& LabHaRT, T. (1998). Development of polarizationopponent interneurons in crickets: Independence of e-vector orientation and strength of polarization? In Göttingen Neurobiology Report 1998, Vol. 2, ed. Elsner, N. \& Wehner, R., p. 416. Stuttgart, New York: Thieme.

Hengstenberg, R., Hausen, K. \& Hengstenberg, B. (1982). The number and structure of giant vertical cells (VS) in the lobula plate of the blowfly Calliphora erythrocephala. Journal of Comparative Physiology A 149, 163-177.

Kral, K. \& Meinertzhagen, I.A. (1989). Anatomical plasticity of synapses in the lamina of the optic lobe of the fly. Philosophical Transactions of the Royal Society $B$ (London) 323, 155-183.

KrapP, H.G. (2000). Neuronal matched filters for optic flow processing in flying insects. In International Review of Neurobiology, Vol. 44, ed. Lappe, M., pp. 93-120. San Diego, California: Academic Press.

Krapp, H.G. \& Hengstenberg, R. (1996). Estimation of self-motion by optic flow processing in single visual interneurons. Nature 384, 463-466.

Krapp, H.G. \& Hengstenberg, R. (1997). A fast procedure to determine local receptive-field properties of motion-sensitive visual interneurons. Vision Research 37, 225-234.

Krapp, H.G. \& EgElHAAF, M. (1999). Local preferred directions of visual wide field neurons and the compound eye geometry of the blowfly
Calliphora erythrocephala. In Göttingen Neurobiology Report 1999, Vol. 2, ed. Elsner, N. \& Eysel, U., p. 440. Stuttgart, New York: Thieme.

Krapp, H.G., Hengstenberg, B. \& Hengstenberg, R. (1998). Dendritic structure and receptive-field organization of optic flow processing interneurons in the fly. Journal of Neurophysiology 79, 1902-1917.

LAughlin, S.B. \& Osorio, D. (1989). Mechanisms for neuronal signal enhancement in the blowfly compound eye. Journal of Experimental Biology 144, 133-146.

McCann, G.D. \& ArnetT, D.W. (1972). Spectral and polarization sensitivity of the dipteran visual system. Journal of General Physiology 59, 534-558.

Miles, F.A. \& Wallman, J. (1993). Visual Motion and its Role in the Stabilization of Gaze. Amsterdam, London, New York, Tokyo: Elsevier

Petrowitz, R., Dahmen, H., EgelhaAf, M. \& Krapp, H.G. (2000). Arrangement of optical axes and spatial resolution in the compound eye of the female blowfly Calliphora. Journal of Comparative Physiology A 186, 737-746.

Rybak, J. \& Meinertzhagen, I.A. (1997). The effects of light reversals on photoreceptor synaptogenesis in the fly Musca domestica. European Journal of Neuroscience 9, 319-333.

Srinivasan, M.V. \& ZHang, S.W. (2000). Analysis of optic flow by the insect visual system. In International Review of Neurobiology, Vol. 44 ed. Lappe, M., pp. 67-92. San Diego, California: Academic Press.

Strausfeld, N.J. (1976). Atlas of an Insect Brain. Berlin, Heidelberg, New York: Springer.

TANAKA, K. \& SAITO, H.A. (1989). Analysis of motion of the visual field by direction, expansion/contraction, and rotation cells clustered in the dorsal part of the medial superior temporal area of the macaque monkey. Journal of Neurophysiology 62, 626-641.

Warren, W.H. \& Hannon, D.J. (1988). Direction of self-motion is perceived from optical flow. Nature 336, 162-163.

Watson, D.F. (1994) Contouring: A Guide to the Analysis and Display of Spatial Data. Oxford, UK: Pergamon Press.

Weber, E. (1970). Grundriss der biologischen Statistik. Stuttgart: Gustav Fischer Verlag.

Wiesel, T.N. (1982). Postnatal development of the visual cortex and the influence of the environment. Nature 299, 583-591.

Wylie, D.R.W., Bischof, W.F. \& Frost, B.J. (1998). Common reference frame for neuronal coding of translational and rotational optic flow. Nature 392, 278-282. 